\title{
Biomechanics: A Frontier Microbial Biotechnology
}

\section{Ashley D Jackson ${ }^{1,2}$ and Matthew BA McCullough ${ }^{1,2 *}$}

${ }^{1}$ Department of Chemical, Biological and Bioengineering, North Carolina Agricultural and Technical State University, Greensboro, USA

${ }^{2}$ Engineering Research Center for Revolutionizing Metallic Biomaterials, North Carolina Agricultural and Technical State University, Greensboro, USA

While cellular system mechanics is an emerging area in need of additional study. Preliminary research suggests that cells grow, respond to and provide mechanical stimuli. This discovery of the impact of mechanics on cellular function has led to a better understanding of human cellular physiology, including phagocytosis, the autoimmune system, and even cancer cell proliferation. Each of these systems relies on mechanical stimuli from contact with other cells or within the cell itself. The capabilities of computational modeling have increased exponentially in the past decade thanks to improved computational resources. The combination of molecular dynamics, quantum mechanics, and computational Nano mechanics has greatly increased researchers' ability to analyze nanoscale systems in a way that uses less resources and time. Perhaps the most challenging aspect of modeling cellular function is the need to operate on multiple temporal and spatial scales [1]. Many of modeling schemes that account for multi-scale space are built on multi-scale homogenization theory. In addition to the aforementioned techniques, immersed molecular electrokinetic finite element uses multi-scale frameworks. Such abilities are able to accurately model RNA binding at a Nano level [2]. This combination is especially powerful as it draws on the expertise of material mechanics to solve problems of material properties within the cell and the cellular membrane. Extensions of this form of finite element analysis have included tight binding, to address crack propagation on a cellular level within bone and could address failure criteria in tissue $[3,4]$. Other cellular modeling examples include the study of red blood cell aggregation [4] as well as single and multi-phase tumors. Such complicated processes associated with tumor cells used thermodynamically consistent mixture models based on Cahn-Hilliard theory [5,6]. Advanced computational models can be developed by observing in vitro behaviors of cells. The beauty of using cell culture is that the researcher can control the environment, changing factors such as temperature, initial amount of cells, $\mathrm{pH}$, growth medium, etc. These factors and behaviors can then be developed into a computational model such as the explanatory models described by Smallwood et al. [7]. Unfortunately many of these computational methods have not been fully applied to microbial behavior mechanisms, and validation of these models is often based upon the agent-based model of cellular interaction [7]; wherein each cell, or agent, is independently governed by a set of rules that account for each behavior in response to real situations [7].

It has been observed that cells will exhibit different conformational changes such as elongation or contraction based on mechanical stimulus like shear stress, as is the case in endothelial cells. Recent examples of the study of such influences include Actomyosin contraction as a key contributor to the shape of cells and tissues during early morphogenesis in Drosophila [8]. The influence of mechanics is also seen in plants as Heisler and colleagues [9] noted that the orientation of two different types of microtubules are dependent upon biomechanical factors. Additional experimental results have shown that the traction force generate by epithelial cells is dependent upon the stiffness or rigidness of the surrounding substrate [10]. Since microbes are single-celled organisms, microbes may respond in the same manner. Underhill and Ozinsky [11] reviewed the relationship between contact, the activation of multiple pathways, and signaling networks within microbes.
These signaling pathways play a key role in defining the phagocyte response. Combining computational and cell experimental methods to predict microbial mechanics will lead to additional discoveries of new disease pathologies. An example of such discoveries is the 3-dimensional model of the HIV-1 virion developed by Johnson et al. [12]. In summary, the fields of biomechanics, microbial research, and computational modeling are advancing at a rapid pace. The overlap of these research areas and the resulting applications will solve some of the most challenging problems facing microbial technology.

\section{References}

1. Bao G, Bazilevs Y, Chung JH, Decuzzi P Espinosa HD, et al. (2014) USNCTAM perspectives on mechanics in medicine. J R Soc Interface 11: 20140301.

2. Li Y (2014) Multiscale modeling and uncertainty quantification in nanoparticlemediated drug/gene delivery. Computational Mechanics 53: 511-537.

3. Abraham FF (1998) Spanning the continuum to quantum length scales in a dynamic simulation of brittle fracture. EPL (Europhysics Letters) 44: 783.

4. Liu Y (2004) Coupling of Navier-Stokes equations with protein molecular dynamics and its application to hemodynamics. International Journal for Numerical Methods in Fluids. 46: 1237-1252.

5. Cristini V, Li X, Lowengrub JS, Wise SM (2009) Nonlinear simulations of solid tumor growth using a mixture model: Invasion and branching. J Math Biol 58: 723-763.

6. Sciumè G, Shelton S, Gray W, Miller C, Hussain F, et al. (2013) A multiphase model for three-dimensional tumor growth. New J Phys 15: 015005.

7. Smallwood RH, Holcombe WM, Walker DC (2004) Development and validation of computational models of cellular interaction. J Mol Histol 35: 659-665.

8. Fernandez-Gonzalez R, Zallen JA (2009) Cell mechanics and feedback regulation of actomyosin networks. Sci Signal 2: pe78.

9. Heisler MG (2010) Alignment between PIN1 polarity and microtubule orientation in the shoot apical meristem reveals a tight coupling between morphogenesis and auxin transport. PLoS biology 8: 2409.

10. Saez A, Buguin A, Silberzan P, Ladoux B (2005) Is the mechanical activity of epithelial cells controlled by deformations or forces? Biophys J 89: L52-54.

11. Underhill DM, Ozinsky A (2002) Phagocytosis of microbes: complexity in action. Annu Rev Immunol 20: 825-852.

12. Johnson GT, Goodsell DS, Autin L, Forli S, Sanner MF, et al. (2014) 3D molecular models of whole HIV-1 virions generated with cellPACK. Faraday Discuss 169: 23-44.

*Corresponding author: Matthew BA McCullough, Department of Chemical, Biological and Bioengineering, North Carolina Agricultural and Technical State University, Greensboro, USA, Tel: +1 336-334-7500; E-mail: mattbm@ncat.edu

Received July 03, 2015; Accepted August 25, 2015; Published September 01, 2015

Citation: Jackson AD, McCullough MBA (2015) Biomechanics: A Frontier Microbial Biotechnology. J Microb Biochem Technol 7: 257. doi:10.4172/1948-5948.1000220

Copyright: (C 2015 Jackson AD, et al. This is an open-access article distributed under the terms of the Creative Commons Attribution License, which permits unrestricted use, distribution, and reproduction in any medium, provided the original author and source are credited. 